\title{
Plasmid-determined Transformation of cis-Abienol and Sclareol in Rhodococcus erythropolis JTS-131
}

\author{
Tadaharu Hieda, Yoichi Mikami and Yukiteru OBI \\ The Central Research Institute, Japan Tobacco and Salt Public Corporation, \\ 6-2 Umegaoka, Midori-ku, Yokohama 227, Japan
}

Received September 1, 1982

\begin{abstract}
cis-Abienol-transformable bacterium JTS-131 was identified as Rhodococcus erythropolis. This bacterium had a plasmid, pCA4. Cured strains which had no plasmid were obtained by mitomycin $\mathrm{C}$ treatment. These cured strains lost the ability to oxidize the C-18 methyl of cis-abienol and sclareol. From these results, it was considered that C-18 methyl oxidation of these labdanes was determined by the plasmid, pCA4, in this bacterium.
\end{abstract}

In the previous paper, ${ }^{1)}$ we reported the microbial transformation of cis-abienol [(12Z)-labda-12,14-diene] and sclareol [labd14 -en-13 $\beta$-ol] by the soil bacterium, JTS-131, together with their transformation pathways.

In other papers, ${ }^{2 \sim 4)}$ we reported that Nocardia restricta JTS-162 transformed cisabienol and sclareol. And, it was considered that C-18 methyl oxidation and A-ring splitting of these labdanes were determined by the plasmid, $\mathrm{pCAl}$, in this strain.

The present report describes taxonomic studies on bacterium JTS-131 and evidence which indicates that the transformation pathways of cis-abienol and sclareol in bacterium JTS-131 may be determined by a plasmid.

\section{MATERIALS AND METHODS}

Microorganisms. Bacterium JTS-131 isolated as a cisabienol-transformable microorganism ${ }^{1)}$ was used in this study. Rhodococcus erythropolis ATCC 4277 was used as the type culture.

Morphological characteristics. Cell form and growth on L-agar, nutrient agar, and rich and poor agars ${ }^{5)}$ were observed after 1 to 7 days incubation. Huckers modification method ${ }^{6)}$ was employed for gram-staining. Motility was investigated by the hanging drop method, and flagellation was confirmed by the staining method of Toda.? The acid-fast test was carried out by the method of ZiehlNeelsen. ${ }^{8)}$ For the milk culture, B.P.C. was used as a pH indicator.
Biochemical characteristics. Production of acid from carbohydrates was observed for 2 weeks at $28^{\circ} \mathrm{C}$, with a medium composed of $3.0 \mathrm{~g}$ of pepton, $2.5 \mathrm{~g} \mathrm{NaCl}, 5.0 \mathrm{~g}$ carbohydrate and $1000 \mathrm{ml}$ distilled water, adjusted to $\mathrm{pH}$ 7.2 and colored with B.C.P.

Assimilation of organic compounds as sources of carbon and energy for growth was examined by the method of Stanier et al..$^{9)}$ In general, a concentration of $1.0 \%$ was used except for sugars and aromatic compounds which were used a concentrations of $0.2 \%$ and $0.025 \%$, respectively. Nitrite formation from nitrate was examined in nitrate and succinate-nitrate broth $^{10)}$ after 1, 3, 5 and 8 days incubation at $28^{\circ} \mathrm{C}$. Extracellular activity was determined after 1,3 and 7 days incubation at $28^{\circ} \mathrm{C}$ with a DNase test medium (Eiken Chemical Co., Ltd. Japan). Urease was examined by the method of Gordon et al. ${ }^{11}$.

Isolation of DNA and determination of GC content. Bacterium JTS-131 was cultured in L-broth containing glycine at a final concentration of $1 \% \cdot{ }^{12)}$ Isolation of DNA was carried out by Marmur's method. ${ }^{13)}$ The DNA base composition was calculated from its thermal denaturation temperature $(\mathrm{Tm})$.

Determination of meso-diaminopimeric acid (meso$D A P)$. Bacterium JTS-131 was cultured in L-broth for 3 days at $28^{\circ} \mathrm{C}$ with shaking. Preparation and hydrolysis of cell walls was carried out by the method of Kurane $e t$ al. ${ }^{14}$ meso-DAP was determined by thinlayer chromatography (TLC). TLC was performed on a cellulose plate with the following system; methanol-distilled water- $6 \mathrm{~N}-\mathrm{HCl}$ pyridine $=80: 26: 4: 10{ }^{15}$ )

Isolation and determination of mycolic acid. Bacterium JTS-131 was cultured in L-broth for 3 days at $28^{\circ} \mathrm{C}$ with shaking. Isolation of mycolic acid from cell walls was carried out by the method of Yano et al. ${ }^{16)}$ After 
trimethylsilylation, the resultant trimethylsilyl esters were analyzed by GC mass spectrometry (Hitachi M-80 GC mass spectrometer) with a glass column $(1 \mathrm{~m} \times 3 \mathrm{~mm}$ i.d. $)$ packed with $1 \%$ Silicone OV-1 on Chromosorb W (AW, DMCS, $80 \sim 100$ mesh).

Media. Medium-A, containing (g/liter) $\left(\mathrm{NH}_{4}\right)_{2} \mathrm{SO}_{4}$ 2.0, $\mathrm{K}_{2} \mathrm{HPO}_{4} 2.0, \mathrm{MgSO}_{4} \cdot 7 \mathrm{H}_{2} \mathrm{O} 0.2, \mathrm{CaCl}_{2} \cdot 2 \mathrm{H}_{2} \mathrm{O} \quad 0.2$, and $\mathrm{FeSO}_{4} \cdot 7 \mathrm{H}_{2} \mathrm{O} 0.01$, and adjusted to $\mathrm{pH} 7.2$ was used as the transformation basal medium. Medium-B, containing sodium succinate $(5 \mathrm{~g} /$ liter $)$ in medium-A, was used as the transformation medium. L-Broth, containing (g/liter) Bacto-trypton (Difco) 10.0, Bacto-yeast extract (Difco) 5.0, $\mathrm{NaCl} 5.0$, and glucose 1.0, and adjusted to $\mathrm{pH}$ 7.0, was used for cell preparation for meso-DAP, mycolic acid and the plasmid. L-Agar, containing $1.5 \%$ of agar in L-broth, was used as the stock medium.

Preparation of L-agar plate containing cis-abienol. An Lagar plate containing uniformly dispersed cis-abienol (CAL-plate) was prepared according to the method of Peterson et al. ${ }^{17)}$ Two grams of cis-abienol in $10 \mathrm{ml}$ of $1 \%$ (w/v) Tween-60, autoclaved for $15 \mathrm{~min}$ at $121^{\circ} \mathrm{C}$ and then cooled to about $70^{\circ} \mathrm{C}$, was added to separately sterilized Waring vessels containing $200 \mathrm{ml}$ of cooled, molten L-agar. After blending for $2 \mathrm{~min}$, the mixture $(5 \mathrm{ml})$ was poured into a sterile plate in which $10 \mathrm{ml}$ of L-agar had already hardened. It was necessary to remove foam from the surface of the plate with a gas burner for a few seconds.

A CAA-plate and CAB-plate were also prepared by the same procedure using medium- $A$ and medium-B, respectively.

Curing experiment. Bacterium JTS-131 was inoculated into a test tube containing $5 \mathrm{ml}$ of $\mathrm{L}$-broth and incubated for $40 \mathrm{hr}$ at $28^{\circ} \mathrm{C}$. The cells were transferred to fresh Lbroth containing mitomycin $C(1.0 \mu \mathrm{g} / \mathrm{ml})$ to a cell concentration of $10^{5} \sim 10^{6}$ per $\mathrm{ml}$ and incubated at $28^{\circ} \mathrm{C}$ until full growth had occurred $\left(3 \sim 4 \times 10^{9}\right.$ cells $\left./ \mathrm{ml}\right)$. After dilution, the resulting culture was incubated on an L-plate. To select the cured strain, single colonies isolated from the L-plate were exmained for the transformation ability using basal medium-A $(5 \mathrm{ml} /$ test tube), containing $0.1 \%$ cisabienol and $0.01 \%$ Tween-60. After $48 \sim 60 \mathrm{hr}$ incubation, the culture was acidified to $\mathrm{pH} 3.0$ and extracted with ethyl acetate. The extract was analyzed by TLC and GC-MS.

Microbial transformation of cis-abienol, sclareol and their transformation products. Compound A-1 [(12Z)labda-12,14-dien-18-ol], compound A-2 [(12Z)-labda12,14-dien-18-oic acid] and compound A-3 [(12Z)-labda12,14-dien-18-oic acid methylester] were obtained as the transformation products from cis-abienol with bacterium JTS-131. ${ }^{1)}$ Compound A-4 [labda-12,14-dien-17 $\alpha$-ol] was obtained as the transformation product from cis-abienol with $N$. restricta JTS-162. ${ }^{18}$ ) Compound S-1 $[13 \beta$ hydroxylabd-14-en-18-oic acid] and compound S-2 [13 $\beta$ - hydroxylabd-14-en-18-oic acid methylester] were also obtained as the transformation products from slcareol with bacterium JTS-131. ${ }^{1)}$ Compound S-3 [labd-14-en-13 $\beta$,18diol] was prepared by the reduction of compound S-1 using $\mathrm{LiAlH}_{4} \cdot{ }^{1)}$ Ten $\mathrm{mg}$ of each compound described above was added to $5 \mathrm{ml}$ of medium-A or medium-B containing the cured strain (about $10^{8} / \mathrm{ml}$ ) and $0.5 \mathrm{mg}$ of Tween-60. After $60 \mathrm{hr}$ incubation, the resulting culture was acidified to $\mathrm{pH} 3.0$ and extracted with ethyl acetate. The extract was analyzed by TLC and GC-MS.

Thin layer chromatography (TLC). The sample was spotted on a Silica gel G plate (Merck) and developed with $n$-hexane-ethyl acetate $(7: 3$ or $5: 5, \mathrm{v} / \mathrm{v})$. After evaporation of the organic solvents, the compounds were detected by spraying $p$-anisaldehyde $(5 \%)$ and $\mathrm{H}_{2} \mathrm{SO}_{4}(5 \%)$ in $90 \%$ ethanol onto the plate and heating it at $120^{\circ} \mathrm{C}$ for $3 \mathrm{~min}$.

Gas chromatography-mass spectrometry (GC-MS). GCMS was performed under the following conditions, using a Hitachi M-80 GC mass spectrometer. The column was a glass tube $(1 \mathrm{~m} \times 3.2 \mathrm{~mm}$ i.d.) packed with $10 \%$ Silicone OV-101 on Chromosorb W (AW, DMSC, 60 80 mesh) or $2 \%$ Silicone XE-60 on Chromosorb W (AW, DMCS, $60 \sim 80$ mesh).

Preparation of crude plasmid DNA. Plasmid DNA for electrophoresis was prepared by the method of Hansen and Olsen. ${ }^{19)}$ Cells grown in L-broth were suspended in $25 \%$ sucrose solution, and lysed by the addition of lysozyme, ethylenediaminetetraacetic acid (EDTA) and sodium dodecyl acetate (SDS) followed by heat pulsing at $55^{\circ} \mathrm{C}$. The clear viscous lysate thus obtained was subjected to alkaline denaturation and then salt-precipitation to remove chromosome-membrane complexes. Plasmid DNA was concentrated with polyethyleneglycol and dissolved in a small volume of buffer containing $50 \mathrm{~mm}$ Tris, $5 \mathrm{~mm}$ EDTA and $50 \mathrm{~mm} \mathrm{NaCl}$ at $\mathrm{pH} 8.0$ (TES buffer).

$\mathrm{CsCl}$-ethidium bromide centrifugation. Further purification of crude plasmid DNA was carried out by centrifugation in $\mathrm{CsCl}$-ethidium bromide solution according to the procedure of Yano et $a .^{20)}$

Agarose gel electrophoresis. Agarose gel electrophoresis was performed according to the method of Kado and $\mathrm{Liu}^{21)}$ in a horizontal apparatus $(13.0 \times 13.5 \times 0.5 \mathrm{~cm})$ of 0.5 and $0.7 \%$ agarose (Sigma type II) at $50 \mathrm{~V}$. The gel was stained with ethidium bromide in water $(0.5 \mu \mathrm{g} / \mathrm{ml})$ for $30 \mathrm{~min}$. The gel was visualized on a transilluminator model C-62 (Ultra-Violet Products Inc., San Gabriel, Calif.) and photographed on Polaroid 667 film, using UV and orange filters. 
Table I. Taxonomical Properties of Bacterium STS-131

Rods and cocci $(0.5 \sim 0.7$ by $5 \sim 15 \mu \mathrm{m}$ after $12 \mathrm{hr}$ incubation, $0.5 \sim 0.7$ by $0.5 \sim 1.5 \mu \mathrm{m}$ in old cultures). Fragmentation begins in the center of a colony after $12 \sim 14 \mathrm{hr}$ incubation. Microcysts are common in old cultures. Gram-positive. Non-motile. Partially acid fast.

Nutrient agar colonies ( 5 days); $0.5 \sim 1.5 \mathrm{~mm}$, pasty and mucoid, pinkish gray with entire margins. Growth restricted on nutrient gelatin; no liquefation.

Acid from D-fructose, D-glucose, glycerol, sorbitol, maltose, mannitol, mannose, sucrose, trehalose and dextrin.

Carbon utilization; D-alanine, L-alanine, D-fructose, D-glucose, glycerol, maltose, mannitol, mannose, malate, lactate, sebacic acid, adenine, inuline, adipate, gluconate, paraffin, sodium acetate, sodium citrate, sodium pyruvate, sodium succinate and trehalose.

Urease present. Phosphatase present. Nitrite not produced from nitrate in nitrate broth. Nitrite produced from nitrate in succinate-nitrate broth.

Litmus milk was alkaline, no coagulation.

Adenine, esculin, and Tween-20, 40 and 60 hydrolysed. Casein not hydrolysed.

Growth temperature; $5 \sim 40^{\circ} \mathrm{C}$. Optimum; $28^{\circ} \mathrm{C}$. Growth $\mathrm{pH} ; 6 \sim 10$. Optimum; 7.5. Not inhibited by $7 \% \mathrm{NaCl}$. Sensitive to penicillin ( 5 i.u. discs.). Lysozyme sensitive. GC content; $63.0 \mathrm{~mol} \%\left(T_{\mathrm{m}}\right)$.

\section{RESULTS}

Taxonomical properties of cis-abienol transformable bacterium JTS-131

The morphological and physiological properties of soil bacterium JTS-131 are summarized in Table I. This bacterium was grampositive, non-endospore forming, rodfilamentous, partially acid-fast, non-motile and aerobic, and had meso-diaminopimeric acid (meso-DAP) in the cell wall. According to Yamada and Komagata, ${ }^{22)}$ and Komura et al. ${ }^{23)}$ meso-DAP as the principal amino acid in the cell wall was found in members of Corynebacterium, Brevibacterium and Nocardia. Furthermore, this strain contained free mycolic acids with 36 to 48 carbon units. It is known that the mycolic acids of Nocardias contain some 50 carbon atoms. ${ }^{24,25)}$ From these results, we tentatively classified this microorganism into the genus Nocardia.

From the results of fragmentation after $12 \sim 14 \mathrm{hr}$ incubation, this strain seemed to fall into "morphological group I of Nocardia," with reference to the 8th edition of Bergey's Manual of Determinative Bacteriology. This bacterium did not hydrolyse casein and produced nitrite in succinate-nitrate broth, but not in nitrate broth. This suggests that the bacterium potentially has nitrate reductase activities as has been reported by Komura et $a l^{26)}$ From other properties summarized in
Table I, bacterium JTS-131 was identified as Nocardia erythropolis (Gray and Thornton) according to the 8th edition of Bergey's Manual. However, in 1980, Nocardia erythropolis was classified as Rhodococcus erythropolis in the "Approved list of bacterial names" presented by the Ad Hoc Committee of the Judical Commission of the International Committee on Systematic Bacteriology (ICBS). ${ }^{27)}$ Bacterium JTS-131 showed the same physiological and biochemical characteristics as ATCC 4277, the type strain of Rhodococcus erythropolis. From these results, we identified bacterium JTS-131 as Rhodococcus erythropolis.

\section{Identification of the cured strain}

To examine whether the transformation of cis-abienol is determined by the plasmid or not, curing experiments were carried out (see Methods). On a CAL-plate and CAB-plate, bacterium JTS-131 could grow as well as on an L-plate, but no clear zones were not formed around the colonies even if the incubation was carried out for $3 \sim 4$ weeks. On a CAA-plate, bacterium JTS-131 could not grow even if the cells were plated from an L-plate by the replica method. Based on these results, the cured strain was obtained by incubation of each colony, using medium-A ( $5 \mathrm{ml} /$ test tube) containing cis-abienol and Tween-60. After $60 \mathrm{hr}$ incubation, the resulting culture was acidified 
to $\mathrm{pH} 3.0$ and extracted with ethyl acetate. The extract was analyzed by TLC and GC-MS.

As a result, three colonies (JTS-13101, -13102 and -13103 ; frequency: 3/430) were obtained on mitomycin $C$ treatment. The three strains thus obtained could not grow on medium-A containing cis-abienol. The concentration of added cis-abienol in the resulting culture was not changed even if prolonged incubation $(\sim 120 \mathrm{hr})$ was carried out. cisAbienol was utilized by the bacterium JTS-131 and not detected in the culture broth after $120 \mathrm{hr}$ incubation under the same conditions.

Microbial transformation of cis-abienol, and compounds A-1, A-2 and A-3 by the cured strains

Compounds A-1, A-2 and A-3 were not utilized by these three cured strains, and the concentration of each compound added to medium-A and medium-B was not changed. The three cured strains slowly utilized cisabienol in medium-B without accumulating transformation products. After $120 \mathrm{hr}$ incubation, the three cured strains had utilized only $12 \%$ of added cis-abienol, though the wild strain utilized all cis-abienol added (Fig. 1). In the presence of EDTA, added to a 12-hr-old culture, the three cured strains could not accumulate any transformation product from cisabienol, but the wild strain accumulated compounds A-1, A-2 and A-3 from cis-abienol in

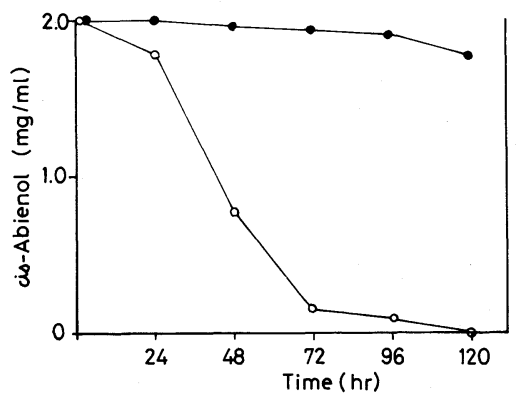

FIG. 1. Time Course of Utilization of cis-Abienol by $R$. erythropolis JTS-131 and its Cured Strain, JTS-13101.

Ten $\mathrm{mg}$ of $c i s$-abienol was added to medium-B $(5 \mathrm{ml} /$ test tube) containing $0.5 \mathrm{mg}$ Tween- 60 and cells. Incubation was carried out at $28^{\circ} \mathrm{C}$ with mechanical shaking.

Symbols: ○— O, JTS-131; - the culture broth. Compound A-4 was utilized by both the wild strain and the cured strains in medium- $\mathrm{A}$ and medium-B.

Microbial Transformation of Sclareol, and compounds S-1, S-2 and S-3 by the cured strains

These three cured strains could not utilize sclareol or compounds S-1, S-2 and $\mathbf{S - 3}$ in medium-A and medium-B. Under the same conditions, wild strain JTS-131 could utilize all these four compounds.

\section{Gel electrophoresis of plasmids}

Figure 2 shows typical agarose gel electrophoresis patterns of plasmids prepared from JTS-131 and JTS-13101. Wild strain JTS131 showed plasmid pCA4. But cured strain JTS-13101 had no plasmid. The other two cured strains also indicated the absence of a plasmid.

Analyses of plasmids in Rhodococcus erythropolis JTS-131 and Nocardia restricta JTS162

To compare the labdane transformation abilities and the plasmids of the two cisabienol transformable bacteria, Rhodococcus erythropolis JTS-131 and Nocardia restricta JTS-162, the plasmids from these two strains were compared in $0.5 \%$ agarose. As shown in Fig. 3, pCA4 was larger than pCA1, pCA2 and pCA3 obtained from $N$. restricta JTS-162.

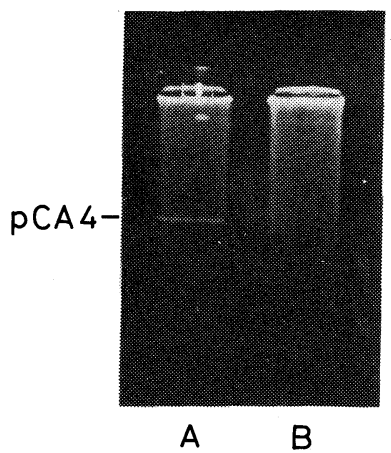

FIG. 2. Agarose Gel Electrophoresis of Plasmids from $R$. erythropolis JTS-131 and its Cured Strain, JTS-13101. A, plasmid of JTS-131; B, plasmid of JTS-13101. 


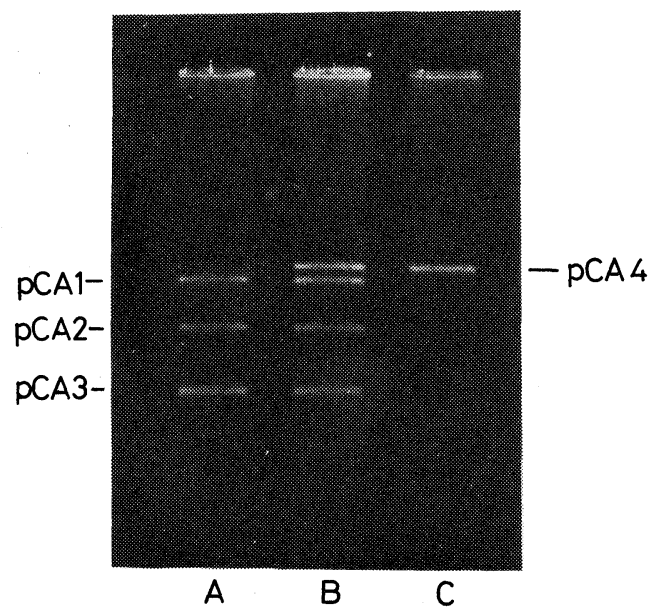

FIG. 3. Agarose Gel Electrophoresis of Plasmids from $N$. restricta JTS-162 and $R$. erythropolis JTS-131.

A, $N$. restricta JTS-162; B, $N$. restricta JTS-162 plus $R$. erythropolis JTS-131; C, R. erythropolis JTS-131.

The DNA bands of these plasmids shown in Figs. 2 and 3 are covalently closed circular (ccc) DNA because of the same mobility on agarose gels as more purified cccDNA on CsCl-ethidium bromide equilibrium density gradients. $^{\text {19) }}$

\section{DISCUSSION}

In the previous paper, ${ }^{1)}$ we reported the microbial transformation of cis-abienol and sclareol by soil bacterium JTS-131. These two labdanes were oxidized by this bacterium and converted to hydroxymethyl, carboxyl acid and methylester derivatives successively.

In the other papers, ${ }^{2,3)}$ we reported that the soil bacterium, Nocardia restricta JTS-162, transformed cis-abienol and sclareol. By the bacterium, the C-8 and C-17 positions of cisabienol were firstly dehydrated, and then the C-18 methyl was oxidized stepwisely and the A-ring was split successively. It was considered that this C-18 methyl oxidation and A-ring splitting were determined by the plasmid (pCA1) in bacterium JTS-162.

In this study, we identified bacterium JTS131 as Rhodococcus erythropolis from the morphological characteristics. This bacterium is widely distributed in soil in Japan as a major

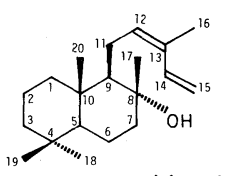

cis-abienol

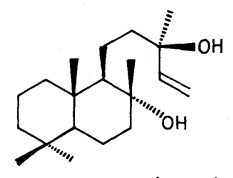

sclareol
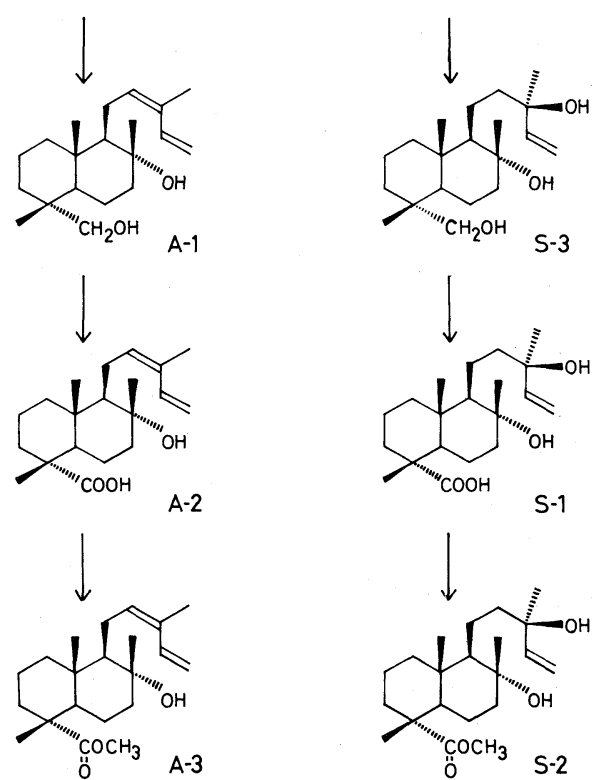

FIG. 4. Plasmid-determined Transformation Pathways for cis-Abienol and Sclareol in R. erythropolis JTS-131.

gram-positive bacterium. ${ }^{28)}$ It was also reported that $R$. erythropolis ( $N$. erythropolis) could metabolize organic compounds such as sterols, ${ }^{29,30,31)}$ aromatic compounds ${ }^{32)}$ and phthalates. ${ }^{14)}$ However, it has not been reported that such abilities to metabolize these organic compounds are determined by the plasmid in $R$. erythropolis ( $N$. erythropolis). In this study, from the results of curing experiments (Fig. 2), it was elucidated that the C-18 methyl oxidation of $c i s$-abienol and sclareol by $R$. erythropolis JTS-131 was determined by the plasmid, pCA4 (Fig. 4). In the cis-abienol transformation by the cured strains from JTS$131,12 \%$ of $c$ is-abienol added was utilized in medium-B after $120 \mathrm{hr}$ incubation (Fig. 1). However, these cured strains could not utilize cis-abienol in medium-A. And they also could not utilize compounds A-1, A-2 and A-3 in both medium-A and medium- $B$. In the previous work, ${ }^{1)}$ it was demonstrated that conversion products from cis-abienol were ob- 
tained only by the addition of metabolic inhibitors, such as EDTA. In this study, these cured strains could not accumulate further conversion products from cis-abienol in the presence of EDTA. These results may indicate that cis-abienol is also utilized by other metabolic pathways different from the transformation pathway previously described. ${ }^{1)}$ The utilization of compound A-4 by this strain may indicate the presence of a transformation pathway containing compound $\mathbf{A - 4}$ as the metabolic intermediate.

The two plasmids, pCA1 and pCA4, which were considered to derermine the $\mathrm{C}-18$ oxidation and A-ring splitting in N. restricta JTS162 and C-18 oxidation in $R$. erythropolis JTS131 , respectively, were analyzed by electrophoresis on $0.5 \%$ agarose gel. As shown in Fig. 3 , the two plasmids showed different mobilities, and pCA4 was larger than pCA1. It is interesting to study the relationship between these two plasmids. Studies on functions and the molecular nature of these two plasmids, pCA1 and pCA4, are now in progress.

Acknowledgment. The authors are grateful to Dr. Akio Seino of Kaken Chemical Co., for his advice for the taxonomic studies.

\section{REFERENCES}

1) T. Hieda, Y. Mikami, Y. Obi and T. Kisaki, Agric. Biol. Chem., 47, 243 (1983).

2) T. Hieda, Y. Mikami, Y. Obi and T. Kisaki, Agric. Biol. Chem., 46, 2249 (1982).

3) T. Hieda, Y. Mikami, Y. Obi and T. Kisaki, Agric. Biol. Chem., 46, 2477 (1982).

4) T. Hieda, Y. Mikami, Y. Obi and T. Kisaki, Agric. Biol. Chem., 46, 3055 (1982).

5) K. Yamada and K. Komagata, J. Gen. Appl. Microbiol., 18, 399 (1972).

6) Society of American Bacteriologists, Committee of Bacteriological Technique (Chairman; M. J. Pelcer, Jr.) "Manual of Microbiological Methods," McGraw-Hill, New York, 1956.

7) T. Toda, Nihon Iji Shimpo, 283, 113 (1928).

8) F. Neelsen, Centr. Med, Wisse., 21, 497 (1883); cited from "Biseibutsu no Bunrui to Dotei (Classification and Identification of Microorganisms)," University of Tokyo Press Co., Tokyo, 1975, p. 218.

9) R. Y. Stanier, N. J. Palleroni and M. Doudoroff, J. Gen. Microbiol., 43, 159 (1966).

10) T. Hasegawa, "Biseibutsu no Bunrui to Dotei (Classification and Identification of Microorganisms)," University - of Tokyo Press, Tokyo, 1975, p. 222.

11) R. E. Gordon and J. M. Mihm, J. Gen. Microbiol., 21, 736 (1959).

12) K. Yamada and K. Komagata, J. Gen. Microbiol., 16, 103 (1970).

13) J. Marmur, J. Mol. Biol., 3, 208 (1961).

14) R. Kurane, T. Suzuki, Y. Takahara and K. Komagata, Agric. Biol. Chem., 41, 1031 (1977).

15) J. L. Stanneck and G. D. Roberts, Appl. Microbiol., 28, 226 (1974).

16) I. Yano and K. Saito, FEBS Lett., 21, 215 (1972).

17) G. E. Peterson, H. L. Lewis and J. R. Davis, J. L. Research, 3, 275 (1962).

18) T. Hieda, Y. Mikami and Y. Obi, Agric. Biol. Chem., 47, 47 (1983).

19) J. B. Hansen and R. H. Olsen, J. Bacteriol., 135, 227 (1978).

20) K. Yano and T. Nishi, J. Bacteriol., 143, 552 (1980).

21) C. I. Kado and S. T. Liu, J. Bacteriol., 145, 1365 (1981).

22) K. Yamada and K. Komagata, J. Gen. Appl. Microbiol., 16, 103 (1970).

23) I. Komura, K. Yamada, S. Otsuka and K. Komagata, J. Gen. Appl. Microbiol., 21, 251 (1975).

24) L. Alshamaony, M. Goodfellow and D. E. Minnikin, J. Gen. Microbiol., 92, 188 (1976).

25) D. E. Minnikin and M. Goodfellow, "The Biology of the Nocardiae," ed. by M. Goodfellow, G. H. Brownell and J. A. Serrano, Academic Press, Inc., New York, N. Y., 1976, p. 160.

26) I. Komura, K. Yamada, S. Otsuka and K. Komagata, J. Gen. Appl. Microbiol., 19, 161 (1973).

27) The Ad Hoc Committee of the Judical Commission of the ICBS, Int. J. Syst. Bacteriol., 30, 356 (1980).

28) K. Kato, Abstracts of Papers, Meeting of the Society of the Science of Soil and Manure, Japan, Kobe, April, 1976, p. 42.

29) D. Kluepfl and C. Vezina, Appl. Microbiol., 20, 551 (1970).

30) S. Sehgal and C. Vezina, Appl. Microbiol., 20, 875 (1970).

31) K. Singh, D. J. Marshall and C. Vezina, Appl. Microbiol., 20, 23 (1970).

32) R. B. Cain and N. J. Cartwright, Biochim. Biophys. Acta, 37, 197 (1960). 